\title{
Synthesis of the algicide bacillamide
}

\section{Valdemar B. C. Figueira, Sundaresan Prabhakar,* and Ana M. Lobo*}

Secção de Química Orgânica Aplicada, Departamento de Química, CQFB-REQUIMTE and SINTOR-UNINOVA, campus FCT-UNL, Quinta da Torre, 2829 Monte da Caparica, Portugal E-mail: sprabhakar@fct.unl.pt; aml@fct.unl.pt

\section{Abstract}

Tryptamine and the mixed anhydride derived from 2-acetylthiazole-4-carboxylic acid and pivaloyl chloride afforded bacillamide.

Keywords: Regiospecific acetylation, bromination, chemoselective oxidation, algicide

\section{Introduction}

Bacillamide, a novel algicide active against the harmful dinoflagellate Cochlodinium polykrikoides, recently isolated ${ }^{1}$ from Bacillus sp. SY-1, was shown by spectroscopic studies to be the amide $\mathbf{1}$ derived biosynthetically most probably from tryptamine (2a) and 2acetylthiazole-4-carboxylic acid (3) (Scheme 1).

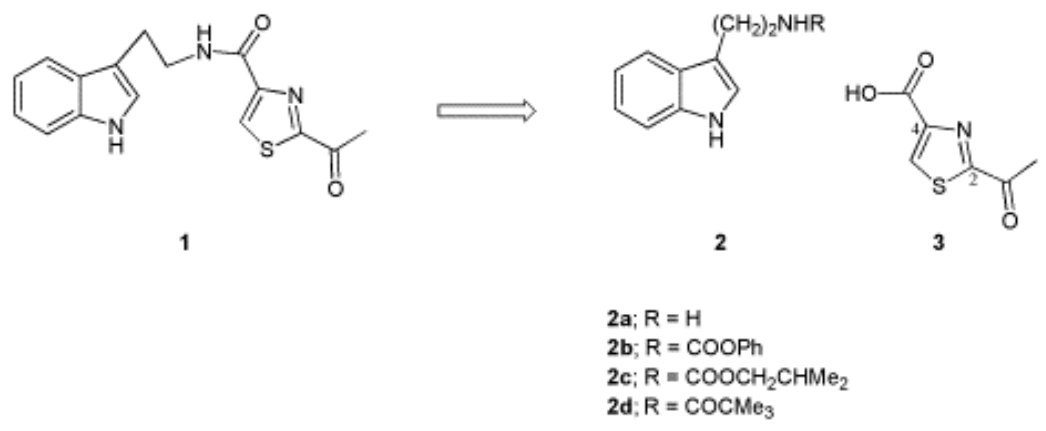

\section{Scheme 1}

As a part of a programme of studies on structure-algicidic properties, a general synthetic method for this class of compounds was deemed desirable. We report herein one such process and describe its application to the first synthesis of bacillamide starting from the commercially available 4-methylthiazole (4). 


\section{Results and Discussion}

A regiospecific introduction of the acetyl group at C-2 in $\mathbf{4}$ was achieved by the procedure described by Dondoni ${ }^{2}$ for unsubstituted thiazole, which involved metallation at C-2 followed by treatment of the resulting C-Li compound with ethyl acetate (Scheme 2). Compound 5a, ${ }^{3}$ thus secured in $64 \%$ yield, on reaction with NBS (2.5 eq) and in the presence of a catalytic quantity of AIBN in $\mathrm{CCl}_{4}$ under reflux, furnished a chromatographically separable mixture of the monobromide $\mathbf{5 b}$ (30\%) (recyclable to $\mathbf{5 c}$ in $50 \%$ yield), the dibromide $5 \mathbf{c}(54 \%)$ and the tribromomethylthiazole 5d (16\%).
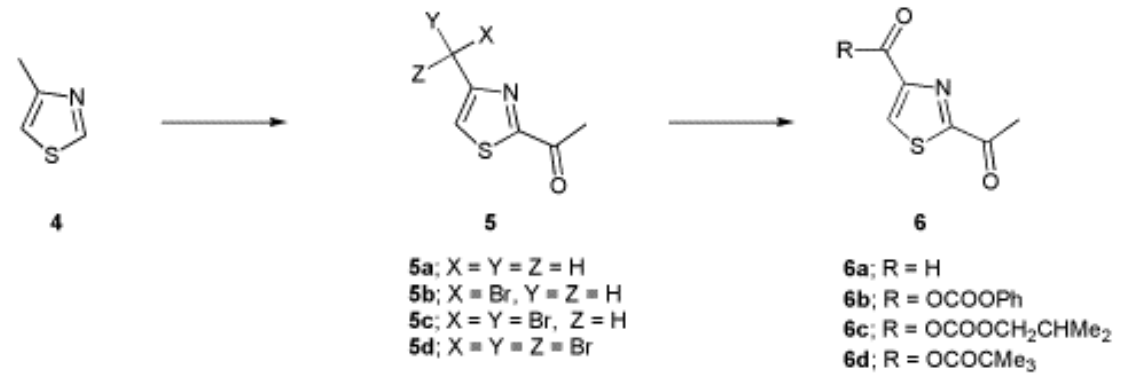

\section{Scheme 2}

Since attempts to convert the monobromide with $\mathrm{NaNO}_{2} / \mathrm{HOAc}$ in $\mathrm{DMSO}^{4}$ or the tribromide directly into the corresponding acid $\mathbf{3}$ by alkaline hydrolysis failed, $\mathbf{5 c}$ was first solvolysed in aqueous acetone in the presence of AgOAc to the aldehyde 6a (82\%) and thence to the requisite acid $3,{ }^{5,6}$ in high yield (94.5\%) by oxidation with $\mathrm{NaClO}_{2}{ }^{7}$

The seemingly simple task of coupling tryptamine $2 \mathbf{a}$ with acid 3 , to form bacillamide in good yield, proved to be unexpectedly difficult. For example, the following three mild methods involving activated carboxylic acid derivatives of $\mathbf{3}$ derived from a) benzotriazole, ${ }^{8}$ b) carbonyl diimidazole, ${ }^{9}$ or c) via Mitsunobu reaction, ${ }^{10}$ all failed to give the requisite product $\mathbf{1}$ in acceptable yields. However, the mixed anhydrides $\mathbf{6 b}$ and $\mathbf{6 c}$ formed in situ from $\mathbf{3}$ and phenylchloroformate and isobutylchloroformate respectively, furnished, on reaction with tryptamine, the title compound $\mathbf{1}$ in low yields (38 and 22\%, respectively). Significant amounts of carbamates of tryptamine $\mathbf{2 b}$ (30\%) and 2c (26\%) were also formed during the reactions indicating that the two carbonyl groups in these mixed anhydrides have similar reactivities towards tryptamine. A markedly improved yield of bacillamide (60\%) was obtained with the use of the mixed anhydride $\mathbf{6 d}$, derived from the sterically more hindered pivaloyl chloride, although the formation of the by product $\mathbf{2 d}$ (28\%) could not be completely suppressed (Scheme 3). The former isolated as a colourless crystalline solid, $\mathrm{mp} 178-180{ }^{\circ} \mathrm{C}$, after preparative tlc, possessed ${ }^{13} \mathrm{C}$ and ${ }^{1} \mathrm{H}$ NMR spectra identical with those of the natural product. ${ }^{11}$ 


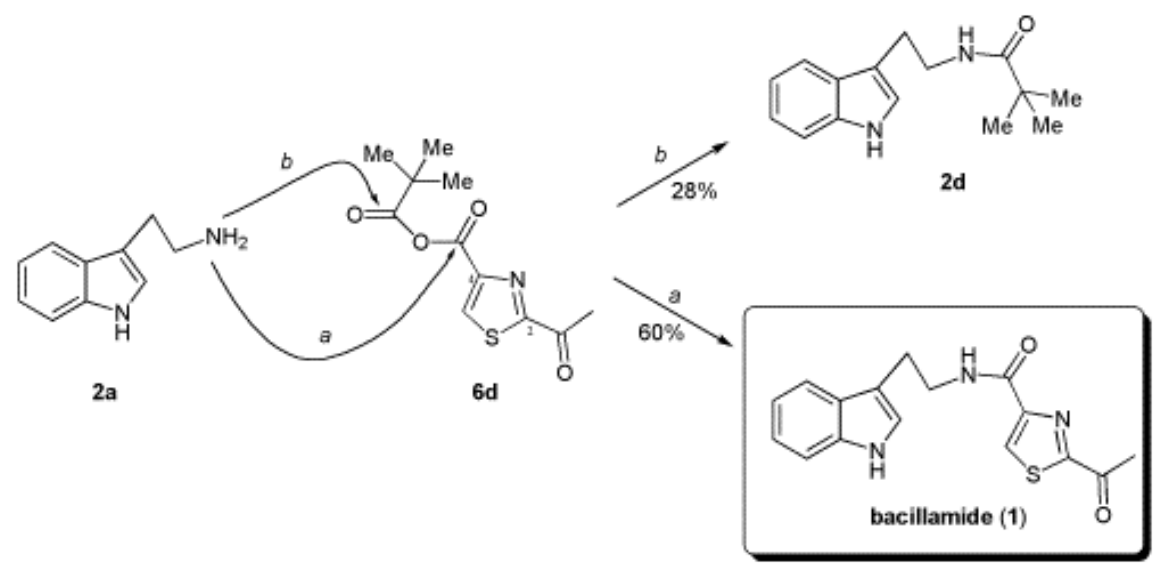

\section{Scheme 3}

\section{Experimental Section}

General Procedures. Melting points were determined with a microscopic hot-stage Reichert Thermovar and are uncorrected. Chromatography was performed using E. Merck silica gel 60 (70-230 mesh). Preparative thin-layer chromatography (PTLC) was performed on plates precoated with silica gel $\mathrm{GF}_{254}(0.5 \mathrm{~mm})$. Infrared spectra (IR) were recorded with a Fourier Perkin-Elmer 157G and 683 infrared spectrophotometers and the frequencies reported in $\mathrm{cm}^{-1}$. Nuclear magnetic resonance spectra $\left({ }^{1} \mathrm{H}\right.$ NMR and ${ }^{13} \mathrm{C}$ NMR) were obtained with a Bruker ARX 400. Chemical shifts are reported in ppm downfield from tetramethylsilane. Mass spectra were obtained on a mass spectrometer GC-TOF Micromass GTC. Elemental analyses were carried out at the Microanalytical Laboratory of the Department. All solvents were purified by standard methods. Petroleum ether refers to fraction boiling between $60-80{ }^{\circ} \mathrm{C}$. Unless otherwise stated work-up is taken to mean drying the water-washed organic extract over anhydrous $\mathrm{Na}_{2} \mathrm{SO}_{4}$ or $\mathrm{MgSO}_{4}$, filtering and evaporating the solvent from the filtrate under reduced pressure.

2-Acetyl-4-methylthiazole (5a). To a stirred solution of BuLi (46 mL, $73.6 \mathrm{mmol}$ ) in dry diethyl ether (100 mL), at $-78{ }^{\circ} \mathrm{C}$ and under an inert atmosphere, was added 4-methylthiazole (4) (6.1 g, $61.5 \mathrm{mmol})$ in dry $\mathrm{Et}_{2} \mathrm{O}(100 \mathrm{~mL})$ dropwise. The mixture after having been kept at $-78{ }^{\circ} \mathrm{C}(1 \mathrm{~h})$ was treated with a solution of dry ethyl acetate $(30 \mathrm{~mL}, 0.306 \mathrm{~mol})$ in dry ether $(100 \mathrm{~mL})$ and then allowed to warm to room temperature. $\mathrm{CH}_{2} \mathrm{Cl}_{2}$ was added (200 $\mathrm{mL}$ ), the solution was washed with aqueous saturated solution of $\mathrm{NaHCO}_{3}$ and the product isolated in the usual manner. The residue thus obtained was purified by column chromatography $\left(\mathrm{CH}_{2} \mathrm{Cl}_{2} / \mathrm{MeOH}, 99: 1\right)$ to provide the title compound 5a, isolated as an orange solid, $5.71 \mathrm{~g}$, (64\%); mp $34{ }^{\circ} \mathrm{C}$ ( $n$-hexane) [lit. ${ }^{3} \mathrm{mp} 35{ }^{\circ} \mathrm{C}$ (n-hexane)]; IR $\left(\mathrm{CH}_{2} \mathrm{Cl}_{2}\right)$ v: 2959, 2933, 2862, 2361, 1690, 1506, $1434 \mathrm{~cm}^{-1} ;{ }^{1} \mathrm{H}-$ NMR $\left(\mathrm{CDCl}_{3}\right) \delta_{\mathrm{H}}: 2.529(\mathrm{~s}, 3 \mathrm{H}), 2.695$ (s, 3H), 7.236 (s, 1H). 
Bromination of 2-acetyl-4-methylthiazole. A mixture of 5a (4.16 g, $24.2 \mathrm{mmol})$, NBS (11.0 g, $61.9 \mathrm{mmol}$ ) and AIBN (62 mg, $0.38 \mathrm{mmol}$ ) in previously distilled $\mathrm{CCl}_{4}(44 \mathrm{~mL})$ was, under a nitrogen atmosphere, stirred under reflux $(5 \mathrm{~h})$. The mixture at room temperature was filtered to remove the precipitate, and the filtrate diluted with $\mathrm{CH}_{2} \mathrm{Cl}_{2}(200 \mathrm{~mL})$ and the organic phase washed with a $25 \%$ aqueous sodium hydrogen carbonate solution ( 2 x 20mL). Usual work-up followed by purification of the residue by column chromatography $\left(\mathrm{CH}_{2} \mathrm{Cl}_{2}\right.$ /petroleum ether, 9:1) provided in decreasing order of elution compounds $\mathbf{5 d}, \mathbf{5} \mathbf{c}$ and $\mathbf{5 b}$.

2-Acetyl-4-tribromomethylthiazole (5d). Yield: 1.46 g (16\%); orange oil; IR $\left(\mathrm{CH}_{2} \mathrm{Cl}_{2}\right)$ v: 3117, 1693, 1446, 1360, 1267, $1143 \mathrm{~cm}^{-1}$; ${ }^{1} \mathrm{H}-\mathrm{NMR}\left(\mathrm{CDCl}_{3}\right) \delta_{\mathrm{H}}: 2.769$ (s, 3H), $8.126(\mathrm{~s}, 1 \mathrm{H}) ;{ }^{13} \mathrm{C}-$ NMR $\left(\mathrm{CDCl}_{3}\right) \delta_{\mathrm{C}}: 25.13$ (C-Br, by DEPT), $25.90\left(\mathrm{CH}_{3}\right), 123.61$ (C5), 159.75 (C4), 167.00 (C2), $191.43(\mathrm{C}=\mathrm{O})$. Anal. Calcd for $\mathrm{C}_{6} \mathrm{H}_{4} \mathrm{NOSBr}_{3}$ : C, 19.07; H, 1.07; N, 3.71; S, 8.41. Found: C, 19.28; H, 0.76; N, 3.58; S, 8.47.

2-Acetyl-4-dibromomethylthiazole (5c). Yield: 3.9 g (54\%); colourless solid; mp 68-68.5 ${ }^{\circ} \mathrm{C}$ $\left(\mathrm{CH}_{2} \mathrm{Cl}_{2} / n\right.$-hexane, 1:4); IR $\left(\mathrm{CH}_{2} \mathrm{Cl}_{2}\right)$ v: 1698, 1501, 1423, 1294, $1182 \mathrm{~cm}^{-1} ;{ }^{1} \mathrm{H}-\mathrm{NMR}\left(\mathrm{CDCl}_{3}\right)$ $\delta_{\mathrm{H}}: 2.723(\mathrm{~s}, 3 \mathrm{H}), 6.796(\mathrm{~s}, 1 \mathrm{H}), 7.919(\mathrm{~s}, 1 \mathrm{H}) ;{ }^{13} \mathrm{C}-\mathrm{NMR}\left(\mathrm{CDCl}_{3}\right) \delta_{\mathrm{C}}: 25.89\left(\mathrm{CH}_{3}\right), 32.39$ $\left(\mathrm{CBr}_{2}\right), 124.82$ (C5), 156.68 (C4), 167.02 (C2), $191.24(\mathrm{C}=\mathrm{O}) ; \mathrm{MS}\left(\mathrm{CI}, \mathrm{CH}_{4}\right): \mathrm{m} / \mathrm{z}=297.85$ $\left([\mathrm{M}+\mathrm{H}]^{+}\right), 221.94,219.93,217.92,177.92,140.02$.

Anal. Calcd for $\mathrm{C}_{6} \mathrm{H}_{5} \mathrm{NOSBr}_{2}$ : C, 24.10; H, 1.69; N, 4.68; S, 10.72. Found: C, 24.30; H, 1.56; N, 4.76; S, 10.90 .

2-Acetyl-4-bromomethylthiazole (5b). Yield: $1.6 \mathrm{~g}$ (30\%); colourless solid; mp 59-60 ${ }^{\circ} \mathrm{C}$ $\left(\mathrm{CH}_{2} \mathrm{Cl}_{2} / n\right.$-hexane, 1:4); IR $\left(\mathrm{CH}_{2} \mathrm{Cl}_{2}\right)$ v: 1689, 1450, 1359, 1276, 1217, $1057 \mathrm{~cm}^{-1} ;{ }^{1} \mathrm{H}-\mathrm{NMR}$ $\left(\mathrm{CDCl}_{3}\right) \delta_{\mathrm{H}}: 2.711$ (s, 3H), 4.616 (s, 2H), 7.637 (s, 1H); ${ }^{13} \mathrm{C}-\mathrm{NMR}\left(\mathrm{CDCl}_{3}\right) \delta_{\mathrm{C}}: 26.01\left(\mathrm{CH}_{3}\right)$, $26.22\left(\mathrm{CH}_{2}\right), 124.95$ (C5), 154.38 (C4), 167.28 (C2), $191.39(\mathrm{C}=\mathrm{O}) ; \mathrm{MS}\left(\mathrm{CI}, \mathrm{CH}_{4}\right): \mathrm{m} / \mathrm{z}=$ $219.94\left([\mathrm{M}+\mathrm{H}]^{+}\right)$, 140.02. Anal. Calcd for $\mathrm{C}_{6} \mathrm{H}_{6} \mathrm{NOSBr}$ : C, 32.74; H, 2.75; N, 6.36; S, 14.57. Found: C, 32.84; H, 2.57; N, 6.57; S, 14.66.

2-Acetyl-4-formylthiazole (6a). To a stirred solution of 5c (4.49 g, $15 \mathrm{mmol}$ ) in acetone-water 2:1 (30 mL), at room temperature and under $\mathrm{N}_{2}$ atmosphere, protected from light, silver acetate (5.71 g, $34 \mathrm{mmol}$ ) was added. After stirring (30 h), the solution was filtered over a pad of celite and the latter washed repeatedly with acetone and methylene chloride. The residue obtained on work-up was purified by column chromatography $\left(\mathrm{CH}_{2} \mathrm{Cl}_{2}\right.$ /petroleum ether, 7:3) to afford the title compound 6a, as a yellow solid, $1.91 \mathrm{~g}$ (82\%); mp 57-59 ${ }^{\circ} \mathrm{C}\left(\mathrm{CH}_{2} \mathrm{Cl}_{2} / n\right.$-hexane, 3:1); IR $\left(\mathrm{CH}_{2} \mathrm{Cl}_{2}\right)$ v: 3119, 2874, 1699, 1687, 1466, 1450, $1360 \mathrm{~cm}^{-1} ;{ }^{1} \mathrm{H}-\mathrm{NMR}\left(\mathrm{CDCl}_{3}\right) \delta_{\mathrm{H}}: 2.772$ (s, 3H), 8.449 (s, $1 \mathrm{H}), 10.110(\mathrm{~s}, 1 \mathrm{H}) ;{ }^{13} \mathrm{C}-\mathrm{NMR}\left(\mathrm{CDCl}_{3}\right) \delta_{\mathrm{C}}: 25.97\left(\mathrm{CH}_{3}\right), 132.21$ (C5), 155.89 (C4), $167.96(\mathrm{C} 2), 184.64(\mathrm{CHO}), 191.33(\mathrm{C}=\mathrm{O})$; $\mathrm{MS}\left(\mathrm{CI}, \mathrm{CH}_{4}\right): m / z=156.01\left([\mathrm{M}+\mathrm{H}]^{+}\right)$. Calcd for $\mathrm{C}_{6} \mathrm{H}_{5} \mathrm{NO}_{2} \mathrm{~S}$ : C, 46.44; H, 3.25; N, 9.03; S, 20.66. Found: C, 46.34; H, 3.06; N, 9.25; S, 20.68.

2-Acetylthiazole-4-carboxylic acid (3). A mixture of $6 a(0.78 \mathrm{~g}, 5.03 \mathrm{mmol})$, aq. $50 \% \mathrm{H}_{2} \mathrm{O}_{2}$ solution (0.3 mL, $5.23 \mathrm{mmol}$ ) and a buffer solution of $\mathrm{NaH}_{2} \mathrm{PO}_{4}(\mathrm{pH} 4.3)(1.6 \mathrm{~mL})$ in acetonitrile $(6 \mathrm{~mL})$, while being stirred at $0{ }^{\circ} \mathrm{C}$ and under $\mathrm{N}_{2}$ atmosphere, was treated dropwise with a solution of $\mathrm{NaClO}_{2}(0.574 \mathrm{~g}, 7.04 \mathrm{mmol})$ in water $(6 \mathrm{~mL})$. The reaction was allowed to proceed for 2 hours. After left to reach room temperature, the reaction mixture was taken to dryness in 
vacuum. The resulting residue was sublimed in high vacuum $(0.2 \mathrm{~mm} \mathrm{Hg})$ providing the title compound 3 as a colourless solid, yield: $0.81 \mathrm{~g}(94.5 \%)$; mp 193-195 ${ }^{\circ} \mathrm{C}$ (lit. ${ }^{5} \mathrm{mp} 193-195{ }^{\circ} \mathrm{C}$ ); IR (KBr) v: 3436, 1686, 1639, 1560, 1415, 1355, $1298 \mathrm{~cm}^{-1}$; ${ }^{1} \mathrm{H}-\mathrm{NMR}\left(\mathrm{CDCl}_{3}\right) \delta_{\mathrm{H}}: 2.638(\mathrm{~s}, 3 \mathrm{H}$, $\mathrm{Me}), 7.578$ (s, $1 \mathrm{H}, \mathrm{D}_{2} \mathrm{O}$ exchange), 8.309 (s, 1H).

2-Acetylthiazole-4-carboxylic acid [2-(1H-indol-3-yl)-ethyl]-amide (Bacillamide) (1). A mixture of the acid 3 (25 mg, $0.146 \mathrm{mmol}$ ) and ethyl di-isopropylamine $(21 \mu \mathrm{L}, 0.161 \mathrm{mmol})$ in $\mathrm{CH}_{2} \mathrm{Cl}_{2}(2.3 \mathrm{~mL})$ was stirred at $0{ }^{\circ} \mathrm{C}$ under an inert atmosphere $(15 \mathrm{~min})$. A solution of pivaloyl chloride $(19.8 \mu \mathrm{L}, 0.161 \mathrm{mmol})$ in methylene chloride $(2 \mathrm{~mL})$ was added dropwise. The mixture was kept at $0{ }^{\circ} \mathrm{C}$ for one hour to yield the mixed anhydride $\mathbf{6 d}$ which was reacted immediately with tryptamine $(23.5 \mathrm{mg}, 0.161 \mathrm{mmol})$ in $\mathrm{CH}_{2} \mathrm{Cl}_{2}(3.5 \mathrm{~mL})$. The reaction was allowed to proceed for an additional $30 \mathrm{~min}$. After left to reach room temperature, the reaction mixture was washed with an aqueous saturated solution of $\mathrm{NaHCO}_{3}(10 \mathrm{~mL})$. Usual work-up afforded a residue that on purification by flash chromatography (ethyl acetate/n-hexane, 1:1) provided the title compound $\mathbf{1}$ accompanied with $\mathbf{2 d}$.

Bacillamide (1). Yield: $27.6 \mathrm{mg}$ (60\%); yellow solid; mp 169-170 ${ }^{\circ} \mathrm{C}\left(\mathrm{CH}_{2} \mathrm{Cl}_{2} / n\right.$-hexane, 1:1); IR $\left(\mathrm{CH}_{2} \mathrm{Cl}_{2}\right)$ v: 3401, 3311, 3106, 2922, 1687, 1660, 1544, 1481, 1456, 1359, $1267 \mathrm{~cm}^{-1} ;{ }^{1} \mathrm{H}-$ NMR ( $\left.\mathrm{CDCl}_{3}\right) \delta_{\mathrm{H}}: 2.690$ (s, 3H, Me), 2.956 (t, $2 \mathrm{H}, \mathrm{CH}_{2}, J=7.5 \mathrm{~Hz}$ ), 3.579 (dd, 2H, $\mathrm{CH}_{2}-\mathrm{NH}, J$ $=7.0 \mathrm{~Hz}, 7.5 \mathrm{~Hz}), 6.972\left(\mathrm{t}, 1 \mathrm{H}, \mathrm{CH}_{\text {indole }}, J=7.7 \mathrm{~Hz}\right), 7.059\left(\mathrm{t}, 1 \mathrm{H}, \mathrm{CH}_{\text {indole }}, J=7.7 \mathrm{~Hz}\right), 7.191$ (s, $\left.1 \mathrm{H}, \mathrm{CH}_{\text {indole }}-\mathrm{CH}_{2}\right), 7.329$ (d, $\left.1 \mathrm{H}, \mathrm{CH}_{\text {indole }}, J=7.7 \mathrm{~Hz}\right), 7.608\left(\mathrm{~d}, 1 \mathrm{H}, \mathrm{CH}_{\text {indole }}, J=7.7 \mathrm{~Hz}\right)$, 8.640 (brs, $2 \mathrm{H}, \mathrm{NH}-\mathrm{C}=\mathrm{O}$ and $\mathrm{CH}-\mathrm{S}$ ), 10.820 (s, $\left.1 \mathrm{H}, \mathrm{NH}_{\text {indole }}\right) ;{ }^{13} \mathrm{C}-\mathrm{NMR}\left(\mathrm{CDCl}_{3}\right) \delta_{\mathrm{C}}: 25.22$ $\left(\mathrm{CH}_{2}\right), 25.69\left(\mathrm{CH}_{3}-\mathrm{C}=\mathrm{O}\right), 39.72\left(\mathrm{CH}_{2}-\mathrm{NH}\right), 111.38\left(C_{\text {indole }}\right), 111.64\left(C_{\text {indole }}-\mathrm{CH}_{2}\right), 118.24$ $\left(C_{\text {indole }}\right), 118.34$ ( $\left.C_{\text {indole }}\right), 120.96\left(C_{\text {indole }}\right), 122.63\left(C_{\text {indole }}-\mathrm{NH}\right), 127.23$ ( $\left.C_{\text {indole }}\right), 130.49$ (HC-S), 136.26 ( $\left.C_{\text {indole }}\right), 151.51(C-\mathrm{CONH}), 159.89(\mathrm{NH}-\mathrm{C}=\mathrm{O}), 166.22\left(C-\mathrm{COCH}_{3}\right), 191.41\left(\mathrm{CH}_{3}-\right.$ $C=\mathrm{O}$ ). Anal. Calcd for $\mathrm{C}_{16} \mathrm{H}_{15} \mathrm{~N}_{3} \mathrm{O}_{2} \mathrm{~S}$ : C, 61.32; H, 4.82; N, 13.41; S, 10.23. Found: C, 61.38; $\mathrm{H}$, 4.90; N, 13.26; S, 10.10.

Compound 2d. Yield: $10 \mathrm{mg}$ (28\%); colourless solid; mp 134-135 ${ }^{\circ} \mathrm{C}\left(\mathrm{CH}_{2} \mathrm{Cl}_{2} / n\right.$-hexane, 1:3) [lit ${ }^{12} \mathrm{mp} 134-135{ }^{\circ} \mathrm{C}\left(\mathrm{CH}_{2} \mathrm{Cl}_{2} / n\right.$-hexane, 1:3)].

\section{Acknowledgements}

We thank Fundação para a Ciência e Tecnologia (FC\&T, Lisbon, Portugal) for partial financial support. One of us (V. B. C. F.) is grateful for the award of a doctoral fellowship from FC\&T.

\section{References and Footnotes}

1. Jeong, S.-Y.; Isida, V.; Ito, Y.; Okada, S.; Muraami, M. Tetrahedron Lett. 2003, 44, 8005.

2. (a) Dondoni, A.; Fantin, G.; Fogagnolo, M. Tetrahedron Lett. 1989, 30, 6063. (b) Dondoni, A.; Fantin, G.; Fogagnolo, M.; Pedrini, P. Tetrahedron 1989, 45, 5141. 
3. For a different procedure for the preparation of 2-acetyl-4-methylthiazole, see: Daigo, K.; Ree, L. J. J. Am. Chem. Soc. 1962, 84, 1962.

4. Matt, C.; Wagner, A.; Mioskowski, C. J. Org. Chem. 1997, 62, 234.

5. Barton, M. A.; Kenner, G. W.; Sheppard, R. C. J. Chem. Soc. (C) 1966, 2115. These authors obtained the acid by $\mathrm{KMnO}_{4}$ oxidation of $d l$-2-( $\alpha$-aminoethyl)-thiazole-4-carboxylic acid which in turn was prepared by a multi-step process.

6. The ubiquitous presence of the acid in organisms such as eubacteria and eukaryotes has led to the suggestion that it could be a co-enzyme, cf.: White, R. H. Experientia 1990, 46, 274.

7. Dalcanale, E.; Montanari, F. J. Org. Chem. 1986, 51, 567.

8. (a) Katritzky, A. R.; Shobana, N.; Pernak, J.; Afridi, A. S.; Fan, W.-Q. Tetrahedron 1992, 48, 7817. (b) Katritzky, A. R.; He, H.-Y.; Suzuki, K. J. Org. Chem. 2000, 65, 8210.

9. Okuzumi, T.; Nakanishi, E.; Tsuji, T.; Makino, S. Tetrahedron 2003, 59, 5603.

10. Brosse, N.; Pinto, M.-F.; Jamart-Grégoire, B. J. Org. Chem. 2000, 65, 4370.

11. Photocopies of spectra were kindly provided by Professor S. Okada, Graduate School of Agriculture and Life Sciences, University of Tokyo, Bunkyo-ku, Tokyo.

12. Atkinson, J. D., Eur. Pat. Appl., GB1 055 413, 1967; Chem. Abst. 1967, 67, 43678. 